\title{
A CONCEPTION FOR IDENTIFYING TRUST SERVICE PROVIDERS IN COLLABORATION CLOUD COMPUTING
}

\author{
Venisha.A, Dr.M.Murali
}

\begin{abstract}
: cloud computing model has proven to be effective and popular in offering the benefits such as virtually limitless storage volume, availability and scalability. Hence the cloud environment is managed by varied trust management models. Choosing the most suitable trust management model by the user is often a difficult task. Since there is multiple cloud services offering different level of performances with same functionalities it becomes hard for the user to detect and select the apt cloud service. Csps tend to offer cloud related services to the cloud users keeping sla in consideration. Depending on sort of csp administration offered the payment by the consumer end, each transaction is being evidently distinguished. Regrettably, since qos (quality of service) is not achieved, sla is not looked upon seriously. In various situations and recommendations, administration presence doesn't fulfill the standards and reliability is compromised. The current research paper deals with the difficulty in choosing qualitative csp (cloud service provider). The proposal is of a methodology to reveal the csps trustworthiness pertaining to a cloud scenario. The various levels listed in this mechanism or methodology being: building up of cloud cluster environment, broker creation, machine agent, feedback provider and trust validation. The mentioned system of trust management gains fewer machine agents, feedback is given by external resources, highly effective in trust computing, cpu's of machine won't be left idle or sluggish. It can be stated that the proposed mechanism yield to be effective and feasible as validated by the performance analysis and experimental outcomes. The outcome demonstrates the effectiveness of the approach concerning services matching. With effective use of trust model specific service can be chosen by the cloud user.
\end{abstract}

Keywords: Cloud service Provider, Broker, Feedback provider, Cloud computing, Service level agreement, Machine agent, Cluster Environment.

\section{INTRODUCTION}

Cloud computing is blooming as an extensively needed technology over past few years, but dealing with many shortcomings too. CSS (cloud service selection) being one amidst them. With existence of multiple cloud services across Internet there may be fake or unreliable one also. Detecting the most suitable cloud service for the user tends to become strenuous. The research work put-forth general review of various cloud service models and analyzes main methodologies and prototypes that effectively handles cloud services trust management. A framework that is generic analytical is proposed for assessing the current trust

Revised Manuscript Received on July 05, 2019.

Venisha.A,Department of computer Science,SRM Institute of Science and Technology, kattangulathur,Tamil Nadu-603203,India

Dr.M.Murali, Department of computer Science,SRM Institute of Science and Technology, kattangulathur,Tamil Nadu-603203,India management research prototypes not only in cloud computing but also other concerning areas by utilizing a group of assessment norms. The Topic concerning cloud based trust management is reviewed too. The concept of Trust management in cloud computing lays down various challenges and there have been researches in recent years proposing multiple approaches to conquer this issue. Inspite of attending and resolving this matter yet there exist numerous trust management related concerns namely personalization, security, identification, integrity, scalability and privacy that are often overlooked and needs attention so that cloud computing can completely gain trust. The CSPs tend to offer cloud based services to the cloud users keeping SLA in The research paper presents and imbibes selection of trustful CSP in cloud environment, relying upon the broker agent into machine agent in cloud environment. Following are the level to be handled: Creation of cloud cluster environment, Broker creation, Machine Agent, Feedback Provider, Trust validation. Lesser number of agents are implied at broker end to implement the suggested low overhead trust computing. Moreover the feedback mechanism is applied to enhance efficiency. This means making use of agent at broker level also additional feedback mechanism from external sources. The research work focuses and resolves the trust management issue within cloud scenario relying upon a group of distributed TSPs

- Trust Service Providers. TSPs are trust agents of some third- party that are independent and being trusted by $\mathrm{CP}$ Cloud Providers, CSPs - Cloud Service Providers and CSUs Cloud Service Users offering the cloud consumers with trust linked services. This proof gives info related to the commitment of service by a CSP with respect to SLA Service Level Agreement and feedback of cloud users. Moreover, the information leads to trust evaluation of CSPs that being subjective and objective in type. Using trust propagation network machine agent performs communication with each other which also allows feedback contributor to gain CSP trust information from rest of the feedback providers. Lastly through method of trust validation blacklist ip and unauthorized users are detected, request parameters are thoroughly validated thereafter using decision tree algorithm trustworthy CSP are being validated within the cloud area. Tests reveal that the framework proposed proves to be comparatively stable and effective in recognizing trustworthy and untrustworthy CSPs.

The survey is categorized as mentioned. Section 2

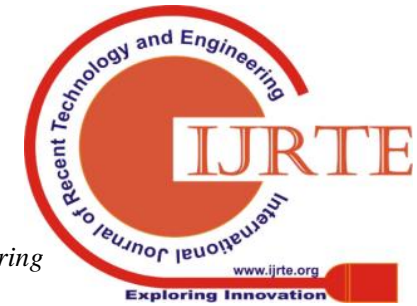


explains background of cloud area, Section 3 briefs regarding work of previous author. Section 4 demonstrates the proposed trust management system and the outlook of various levels. Section 5 proposes future research work and finally concludes the paper.

\section{BACKGROUND}

\section{Cloud types:}

\section{A. Public cloud :}

The cloud framework is owned by companies giving cloud services which are made accessible to enormous people and huge industries. The public clouds grant services to the users via resources using Internet connectivity with an amount according to pay-per-usage. Based on need and requirement the user can extend their usage without actually buying any hardware for using the service. Public cloud providers maintain and offer the infrastructure, providing well enough resources necessary for the cloud users. Usually public clouds are owned by third party firms and offer services to general users and industries at big scale.

\section{B.Private Cloud:}

Private clouds are specifically created for a particular private business. It's a better idea for companies to initially begin with private cloud computing. It offers services to the companies like hosting applications, development environments, and cloud infrastructure alongside handling situations concerning data control and security existing in cloud scenario.

\section{Hybrid Cloud}

The hybrid cloud affirms to be the best combo. Herein the comfort zone of a private cloud is merged with the flexibility and versatility offered by the public cloud. According to the applications need the Hybrid Cloud either utilizes public clouds or Virtual Private Clouds that are hosted off-site which in turn is combined with internal private clouds yielding high security application domain, gaining benefit of both the clouds.

\section{Cloud services:}

Technically there are three models on which CSs relies upon viz Software being the service, service in terms of platform and Infrastructure being the service. According to the applications needs and users demand these services are imparted. The CSPs (cloud service providers) design and work with three distinct layers to actualize diversified technologies in cloud computing, these layers being:

Infrastructure as a service: The management task and cloud resources storage is managed at this level. Since cloud functions on virtual resources, users are granted access to multiple virtual resources viz. software, hardware, servers, etc. in an extent to satisfy application's requirement. .

Platform as a service: The Applications and Software's are built up at this next level facilitating deployment and management of user's application. Software and hardware tools offered by service providers are also included accommodating application frameworks thereby supporting Software as a Service.
II. SOFTWARE AS A SERVICE: THE ClOUd USERS BE ABLE TO ACTUALLY COORDINATE WITH THE APPLICATION AT THIS LEVEL AS HIGH CLASS SERVICE IS OFFERED AT THIS LEVEL ELIMINATING THE REQUIREMENT OF

installing software and hardware at users end. Also there is no need to pay attention on managing service and infrastructure.

\section{DESIGN OF SURVEY}

Nivethitha Somu et al, (2017)[1] has presented that within a public cloud market, detecting the most suitable cloud service amidst a whirlpool of cloud services in accord with user's need tends to become a strenuous task. An appropriate frame-work of service selection aids the client in picking up the desired CSP alongside encouraging the Cloud Service Provider to fulfill the promised SLA -Service Level Agreement thereby enhancing the QOS - Quality of Service.

Disadvantages: Service selection framework produces indefinite CSP ranking as it randomly designates weight assignment to attributes of QoS, replacing the lost values with randomly generated values etc.

Algorithm used: Following two algorithms i.e, HGCM Hypergraph based Computational Model and MDHP Minimum Distance-Helly Property is proposed for CSPs ranking.

Parameters: Dataset relied on QoS attributes is utilized by multiple researchers along with synthetic dataset.

Advantages: The outcome of MDHP, considering various set of study cases affirms that the ranking algorithm holds effective and scalable.

Conclusion: To designate weights to the attributes Maximization (EM) algorithms were imbibed thus reducing the ranking models complexity.

XiaogangWang et, al, (2015)[2]In the area of cloud computing CSS(Cloud service selection) has gained immense limelight. Amidst existence of massive cloud service providers in a unstable cloud environment, selection of appropriate services by the user pertaining to their applications need becomes a strenuous affair, all the more for real time applications.

Disadvantages: The service selection models yields in increasing computation cost, inferior quality and high processing time.

Algorithm used: An adaptive learning methodology that dynamic is imbibed for CSS (cloud service selections) displaying adaptive features.

Parameters: Here the model that is proposed is CSS cloud service selection model that takes into account the cloud service brokers.

Advantages: With this methodology the cloud service selection is optimized dynamically thereby offering the user with quality service result. To help the user effectively choosing the apt cloud service, a cloud service brokers depend on CSS (cloud service selection) model is being provided.

Conclusion: The proposed system reveals enhanced efficiency and performance.

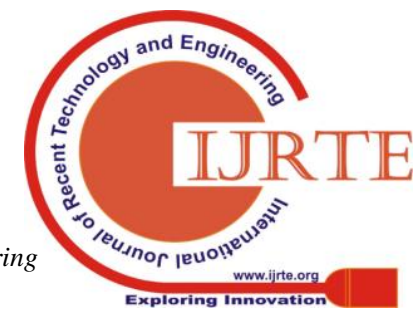


NivethithaSomuet, al (2018)[3] refers an assured research method in Cloud service selection providing wise resolution utilizing ranking service taking into consideration the QoS-Quality of Service features so that trustful CSPs -Cloud Service Providers can be identified amidst a broad spectrum of CSPs that are similar in functions.

Disadvantages: service level ranking is quiet low and inappropriate for CSP selection as fewer parameters are considered.

Algorithm used: HBFFOA - Hypergraph - Binary Fruit Fly Optimization based service ranking Algorithm which is centered solely on trust methodology to identify appropriate trustful CSPs (cloud service providers).

Parameters: Dataset being synthetic Quality of Service derived from WSDream\#2.

Advantages: Function being utilized in HBFFOA is mutational probability which aids in discarding local optima. The WSDream\#2 dataset is used to express the functionality. Detection of CSPs satisfying user's needs, data credibility, service ranking, etc.

Conclusion: WSDream\#2 Synthetic QoS dataset signifies that HBFFOA being effective, practical, scalable and better computational feature as compared with current service selection schemes respect to statistical test, precision, stability and analysis concerns time complexity.

Zhu et al. (2015)[4] Significance of Qualities of Service (QoS) attributes lies in performing trust assessment in cloud computing. Talking about real-time applications, various sorts of cloud services are being present in cloud market space.

Disadvantages: Choosing a suitable sensor network provider that's nodes independent.

Algorithm used: A new system being proposed for integration of wireless sensor network and cloud computing and i.e, ATRCM - Authenticated Trust and Reputation Calculation and management which offers three functions.

Parameters: sensor nodes imbibing this author.

Advantages: Success achieved in providing authenticated services to the sensor network providers and CSPs.

Conclusion: The cloud service users can choose an appealing and satisfying cloud service provider also helping them in determining suitable sensor network provider.

PeiYun Zhang et, al.(2018) Cloud computing has immensely gained a mandatory stand in commercial world with its scientific computing. But the technology, handling cloud resources and data is being chased by numerous trust related issues.

Disadvantages: Not being confidential enough and retrieving Quality-of-Service (QoS) history of cloud services within separate time intervals.

Algorithm used: To reduce the overhead in trust management and enhance unauthorized node detection there is proposal of trust system along with a linked algorithm relying upon domain partition

Parameters: used are related to QoS attribute datasets.

Advantages: A structure along with a methodology used for computing quality and prioritizing Cloud services. Utilizing this framework a remarkable effect is created which will further lead and encourage all the CSPs to stand among the best by competing among each other at the same instance fulfilling their own SLA and enhancing Quality of Service factor.

Conclusion: The proposed methodology reflects overall low performance and high efficiency.

Matin Chiregi et.al [6](2017), have studied previous trust evaluating cloud based mechanisms, the state of the art being one among them. Thereafter examined and compared them with number of key factors that are: dependence, integrity, reliability, confidentiality, security, scalability, safety and dynamicity thus presenting an advice related to the research.

Algorithm used: SLR - Systematic Literature Review and state of the art mechanism.

Advantages: 224 articles are identified, which are brought down to 28 which are the primary ones using the article selection method.

Disadvantage: Suffers from two drawbacks, firstly, the study analyzing the articles were restricted to mere three online databases and Google scholar. Secondly, publications that were non-english were not included in the study.

Conclusion: The survey aims to straight away provide a guidance to the professionals, academicians and researchers so that they can comprehend the trust mechanism realted changes by putting forward the challenging issues and state of the art information.

Erdal Cayirci et.al [7](2018), proposes two models for federated cloud services namely, joint trust and risk model. These models consider the history of performance of the CSPs. It relies on trustworthy third parties to attend the issues linked with consumer and provider so as to gather hard and soft trust related data values thereby granting spontaneous risk monitoring within the cloud.

Algorithm used: Monte-Carlo simulation methodology Advantages: The Monte-Carlo simulation technique is applied for three reasons: for better overview of the models; examining connection amidst engineering related independent variables that (like slope $(\gamma)$, period length and freshness $(\omega)$ ) and dependent variables (like service risks, confidence intervals for security and privacy) and primarily for model verification.

Disadvantage: Inter and Intra data center being used giving fake data to the cloud service provider.

Conclusion: Both Negative and Positive behavior related to performance are detected and differentiated and the historic data's freshness is taken up in this model.

M. H. Ghahramani et.al [8] (2017) Provisioning without or with minimum managerial attempt it becomes easier for the user to fetch from pool of shared computing resources. In a cloud environment, Service provisioning relies upon SLA depicting negotiated contract among consumers and providers.

Algorithm used: Service level agreements (SLA).

Advantages: In depth insight being taken up in 
QoS issues by analyzing technical info related to QoS metrics and frame a comprehensive taxonomy to classify them. The resource allocation problem in cloud computing with varied set of techniques is being tackled.

Disadvantage: Taxonomy does not define the designing of a comprehensive taxonomy.

Conclusion: A summarized survey being conducted keeping in account the proposed Literature model considering the implementation principles to fulfill the promised Quality of Service concerns.

XIANRONG ZHENG et.al [9](2017), referring specific cloud services, a method of collaborative filtering is proposed which utilizes the Spearman coefficient, on the other hand arguing for proposing cloud services that being QoS oriented. Both QoS ratings and rankings for cloud service are being predicted by applying this approach.

Algorithm used: Collaborative filtering approach, Ranking- based CF approach.

Advantages: $\mathrm{CF}$ approach that is ranking-based when implemented can predict ratings as well as rankings for cloud services with the help of Spearman coefficient.

Disadvantage: The Collaborative filtering is not that secured and is applied to construct recommender systems only. Conclusion: The proposed approach can gain more valid rankings, but few precise ratings with respect to the collaborative filtering approach utilizing the Pearson coefficient.

Hadeel T. El Kassabi et.al, [10](2017) has a MDTM multi-dimensional trust model where there is work-flow evaluation concerning Big Data amidst multiple clouds. ACSP is trustworthy or not is computed in terms of the most recent potential of cloud resources, the proof of reputation being evaluated by adjoining users, history of personal experiences tracked down along with $\mathrm{CP}$.

Algorithm used: MDTM - multi-dimensional trust model related to Big Data, Quality of Cloud Service (QoCS).

Advantages: Corporate sector and Industries making use of Big Data to fetch quality and essential information timely without doubt raise the opportunity of attracting more customers, improvising their operations, reducing their costs and consequently yielding higher profits.

Disadvantage: Various critical functional and non functional needs are supported via Trust model that does not promise trustful trust evaluation.

Conclusion: Collects varied trust components, affirms high QoCS and adequately accustom with the clouds dynamic nature.

Sarbjeet Singh et.al, (2916)[11] trustworthiness is stated as the compliance degree of a CSP to meet the guaranteed quantitative features of Quality of Services that being mentioned in the Service Legal Agreement. With the existence of multiple CSPs lending equivalent services in the cloud computing environment, the Cloud Clients (Ccs) find it strenuous in detecting and differentiating among trustworthy CSPs

Disadvantage: With the existence of multiple CSPs lending equivalent services in the cloud computing environment, the Cloud users find it strenuous in detecting and differentiating among trustworthy and non trustworthy
CSPs

Advantage: Cloud Clients identifying the CSPs trustworthiness from various angles. Systems like this immensely help clients in selecting best suitable CSP amidst wide available options of CSPs.

Algorithm used: CMTES - Compliance-based Multidimensional Trust Evaluation System

Conclusion: The CMTES algorithm provides stability and effectiveness in distinguishing among CSPs that are trustworthy and the one that are untrustworthy. The validity of Compliance- based Multi-dimensional Trust Evaluation System is performed and achieved by utilizing the synthetic data as the standardized dataset is unavailable, furthermore its application is presented on basis of a case study incorporating real time cloud data.

Talal H. Noor [12] et.al, The concept of Trust management deals with various challenges for the development of cloud computing environment. Since sensitive information flows among consumers or users or and TMS - trust management service maintaining privacy of consumers' becomes a tedious task. Design and implementation of Cloud Armor is demonstrated with a framework that is reputation- based trust management which lends a functionalities list in offering TaaS -trust as a service offering low dependability and scalability.

Disadvantage: Unauthorized users may negatively affect a specific cloud user by giving misleading fake feedback and deceive users in believing untrustworthy cloud service providers by building up a number of accounts randomly and providing deluded feedbacks

Advantage: The trust feedbacks reliability and safeguarding users privacy is ascertained by using novel protocol ii) To compute reliability of feedbacks this forms a powerful and secure model thus safeguarding CSPs from unauthenticated users alongside this, various trustworthy CSPs can also be distinguished and iii) Acts as an availability model which maintains the accessibility of the decentralized application of TMS - trust management service

Algorithm used: Cloud Armor, a TMS that is reputation-based.

Conclusion: The feedback of cloud service users' forms the input in assessing the comprehensive trustworthiness concerning cloud services. The outcome of the methodology portrays the applicability of the proposed scheme along with the ability of identifying malicious acts. Zheng et.al, (2013) [13] proposes a comprehensive study regarding delivery of precise QoS ranking for the cloud services. Though ranking-oriented techniques can be utilized to perform optimal CSS among a group of candidates that being functionally similar, they usually avoid any changes concerning the QoS. With the help of these two methods i.e, Rating-oriented collaborative filtering and ranking-oriented collaborative filtering the user can estimate missing $\mathrm{QoS}$ value though the dynamic QoS properties were not considered in MCC, leading to detection of fraud rating.

Disadvantage: consumes enough time and are costly 
real-world service invocations.

Advantage: previous experiences of consumers that have used the service.

Algorithm used: Two personal Quality of Service prediction ranking methodologies being suggested to directly estimate the rankings of Quality of Service.

Conclusion: Utilizing real-world QoS data, experiments are conducted employing 300 users being distributed and 500 being actual Web based services worldwide. The outcome of the experiment reveals that the proposed methodologies beats rest of the existing approaches.

Rajendran and Swamynathan et,al. (2015) To analyze trust dynamics within cloud environment a hybrid model is being proposed. In accord with feedback retrieved from cooperative user there pupation is being computed. The rating of on their trust value thereafter top-k cloud services are advised to the user. The advantage being that it delivers appropriate security, reliability, and dynamicity, but drawback being that it deals with low dependability, confidentiality and safety [14].

Disadvantage: Identifying a trustworthy and reliable service provider is bleak.

Advantage: trust value being computed relying upon compliance and reputation trust. Gathering user feedback collectively, the reputation is being measured.

Algorithm used: Hybrid Model

Conclusion: Enhanced and effective service-selection process concerning cloud environment is depicted by the results.

Charband and Navimipour et, al, (2016) proposes an approach to identify unrealistic feedback within the cloud trust management systems utilizing the feedback assessment component and Bayesian game model. These both new methodologies were introduced so that fake feedbacks could be identified. Though enhanced security and reliability were achieved via this mechanism, but it also resulted in low scalability and dependability [15].

Disadvantage: No such comprehensive and systematic study and analyses exists for these essential methodologies.

Advantage: A systematic and explanatory study and survey of the state-of-the-art knowledge sharing methods being offered within online environments.

Algorithm used: systematic literature review (SLR) concerning online knowledge sharing literatures at the end of 2015.

Conclusion: The outcome delivering state-of-the-art information, this survey aims to straight away reinforce academics and practicing professionals in their comprehension of growth linked to online knowledge sharing mechanisms and methodologies.

Sidhu and Singh et, al, (2016) proposes an effective technique of TOPSIS based trust evaluation frame-work which defines trustworthiness of CSPs. To achieve trust factor on a service provider, at first the conformity values are computed thereafter they are processed utilizing the approach of order of preference of trust, on the basis of likes. The experiments and case study confirms the applicability and usability of the proposed framework. Investigations utilizing the real time cloud data reveal that the framework suffers from low confidentiality [16].

Disadvantage: With existence of numerous cloud services offering equivalent services, it turns out to be strenuous and complicated for the cloud clients (CCs) to distinguish between these CSPs(cloud service providers)

Advantage: The cloud service providers that being trustworthy delivers services in norm with the SLA.

Algorithm used: The system of trust evaluation is framed that utilizes the compliance monitoring approach in measuring CSPs trustworthiness.

Conclusion: With experimental outcome it's proved that in actual cloud scenario the methodology that is put forth can be well implemented thereby computing trustworthiness of Cloud Service Providers by applying their actual time monitoring services.

Bahador Shojaiemehr et.al (2018) proposes market place that being dynamic competitive and conflicting necessities of trading parties. The need arises of proposing automated negotiation mechanisms that abide by the agreements, delivering maximum utilities for both trading parties. The existing article study comprises of CCSN models. Few queries will be put forward; answering these queries will aid the researchers in building up additional effective models in near future. Statistics lists down the existing defects that ought to be administered in future research works [17].

Disadvantage: it takes into account a specific time period in the cloud environment.

Advantage: (1) examining CCSN workings to comprehend open challenges and problems thus offering negotiation systems within cloud environments, (2) detecting productive features concerning CCSN which can yield in increased advantage for the consumers and providers and (3) Highlighting any drawbacks in the existing work thus aiding the researchers enhance the CCSN systems for upcoming researches in future.

Algorithm used: CCSN - Cloud Computing Service Negotiation models, techniques, protocols, frameworks and strategies suggested by the Cloud Computing Service Negotiation models researches.

Conclusion: Examining the outcome of CCSN studies depict that that the researchers ought to frame a negotiation methodology for cloud service negotiation issue that is effective and compatible concerning needs of the consumers and the providers.

\section{SUGGESTED WORK}

\section{Overview}

Cloud computing deals with intensive hurdles like security, privacy, and trust. Amides these challenges, establishing trust among the cloud members remain a prime concern that affects utilizing the cloud services extensively. Fundamentally, the Cloud Service Providers stockpile customer's data to which the consumers possess minimum control for the way their data is maintained. What remains of primary attention is assessment of varied cloud services thereby aiding the cloud users in selection of the right and appropriate service

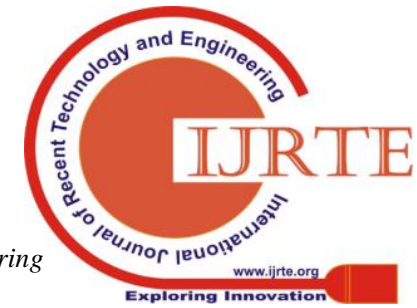




\section{A CONCEPTION FOR IDENTIFYING TRUST SERVICE PROVIDERS IN COLLABORATION CLOUD COMPUTING}

according to fulfill their needs and ensuring that cloud providers grant this service with superior quality. Earlier research work discusses about CSPs trustworthiness during selection process but it suffers from few shortcomings in delivering CSPs that are trustworthy to the users. It's mandatory to retain max trust in the cloud service. Present approach utilizes traditional trust worthy resource matching method that administer massive data volume also simultaneously using every agent within the server leading to increased overhead issue. This may result in reduced efficiency and in drenching of CPU utilization for the existing resources. Resultant, there does not occur any feedback mechanism for the remaining resources. By analyzing earlier paper works, the prime concern that surfaces is identification of trustworthy CSP (cloud service provider). The approach being proposed utilizes minimum machine agent at the broker level furthermore utilizing the feedback mechanism to enhance the efficiency. Performance analysis being applied to validate feasibility and efficiency alongside experimental the cloud environment and to the entities involved. Applying agent at broker level, feedback mechanism from external sources are appended and gain minimum amount of machine agents feedback accepted from external resources. Trust computing is made effective also the Machine CPU's are not left idle or wasted. Following are the mechanisms proposed in the paper to attend various levels: 1. Cluster Environment, 2. Broker Creation, 3. Machine agent, 4. Feedback provider, 5. Trust Validation.

\section{A.Cluster Environment}

Cluster being a collection of various server instances, spreading over greater than single node, with all executing similar configuration. AWS forms the cloud computing provider. In True cloud computing such a service becomes the best example. Account creation is done in AWS and EC2 to introduce either too many or too few virtual servers as per the need in EC2. Cluster formation and application deployment performed in EC2 cluster.

\section{B. Broker}

Cloud Service Brokers (CSBs) surfaced in the picture and ended necessary as there exist heterogeneous amount of both public cloud providers and private cloud providers, along with the necessity to handle the requirements of concerned services pertaining to an enterprise. Brokering grants varied cloud service provisions namely, database, computation, storage, application, etc., as and when consumer places a request. Generally, Broker implies Service discovery, Service intermediation and Service aggregation. It can be generalized as an application forming a bridge among the client and machine agent. EC2 instance makes use of the broker application. Moreover, machine agent rules are being imbibed in this application which behaves somewhat as a proxy.

\section{C.Machine Agent}

Machine agent being a software entity which actually is an independent software program executing on network user's behalf. These agents basically interact among each other, exchange information and aids in executing complicated tasks. Also they are being framed to function in a dynamically altering environment. Managing all the servers is the prime responsibility of Machine agent along with providing rules to outcomes. Trusted cloud computing can be generated within

the broker application related to security concerns like SQL injection, Denial of service, Flooding attack, blacklisted IP.

\section{Feedback Provider}

Trust can contribute to rating-based (or reputation- based) feedback mechanism that turns to be essential reference for rest of the users. Various cloud computing environments relies upon the dependability of a feedback mechanism. An open cloud environment may incorporate a huge amount of untrusted (or malicious) users. Feedbacks and Ratings given by such untrusted users can result in erroneous computed results. The role of Feedback provider is to detect frequent blacklist IP and white list IP using abnormal text in request form thereby allowing the authenticated users only to access the server.

\section{D.Trust Validation}

The Trust methodology exhibits an effective remedy for bettering the safety measures in a cloud computing environment. Generally, the obvious and critical matter being absence of trust amidst cloud users and CSPs cloud service providers. Hence to construct a safeguarded cloud environment, trust plays a mandatory factor. Trust being considered as a subjective mutual relation amidst two parties that can willingly work reliably, securely and dependably in any condition offered for a period of time. Decision tree algorithm is implemented for trust validation wherein a set of genuine parameters are validated and blacklisted IP along with unauthenticated users are identified. This turns the trust computing scheme appropriate enough for wide range of cloud computing environment

\section{CONCLUSION}

With extensive popularity it's observed that equivalent services are being offered across the Internet. QoS forms the essential differentiator factor amidst functionally equivalent services. The current paper offers energy effective mechanisms which aids in picking out the trustworthy CSP(cloud service provider) for cloud users. The proposed trust management system requires minimum amount of machine agents, Feedback is retrieved from external resources, highly effective in trust computing, Machine CPU's are not left idle or wasted. The experimental outcome reveals that our methodology is highly effective in services matching. The Trust model aids the user in choosing the appropriate service effectively.

\section{REFERENCE}

[1] Nivethitha Somu, Kannan Kirthivasan, Shankar Sriram V.S (2017), "A computational model for ranking cloud service providers using hypergraph based techniques", Future Generation Computer Systems, vol. 68, pp. 14-30, Elsevier.

[2] XiaogangWang JianCao YangXiang (2014), "Dynamic cloud service selection using an adaptive learning mechanism in multi-cloud computing", Journal of Systems and Software, Vol. 100, pp. 195-210, Elsevier.

[3] NivethithaSomu Gauthama RamanM.R KannanKirthivasanShankar SriramV.S (2018), "A trust centric optimal service ranking approach for cloud service selection", Future Generation Computer Systems, Vol. 86, pp. 234-252, Elsevier.

[4] Zhu, C., Nicanfar, H., Leung, V. C., \& Yang, L. T (2015),

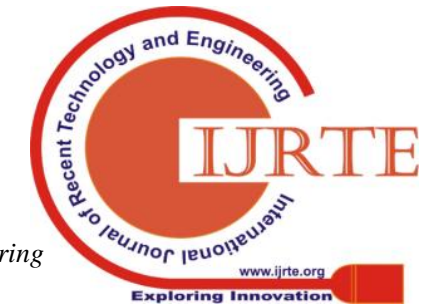


"An authenticated trust and reputation calculation and management system for cloud and sensor networks integration", Information Forensics and Security, IEEE, vol. 10, Issue. 1, pp. 118-131.

[5] PeiYun Zhang, Yang Kong and MengChu Zhou (2018), “A domain partion

- based trust model for unreliable cloud”, IEEE, pp. 2167-2178.

[6] Matin Chiregi, Nima Jafari Navimipour (2017), "Cloud computing and trust evaluation: A systematic literature review of the state of the art chanisms", Electronics Research Institute (ERI), Elsevier.

[7] Erdal Cayirci, Anderson Santana de Oliveira (2018), "Modelling trust and risk for cloud services", Journal of Cloud Computing: Advances, Systems and Applications.

[8] M. H. Ghahramani, MengChu Zhou, Chi Tin Hon (2017), “Toward Cloud Computing QoS Architecture: Analysis of Cloud Systems and Cloud Services", Journal of Automatica Sinica, vol. 4, no. 1, p.p. 6 - 18, IEEE/CAA

[9] Xianrong Zheng, Li Da Xu, Sheng Chai (2017), "QoS Recommendation in Cloud Services”, IEEE, pp. 5171-5176.

[10] Hadeel T. E Kassabi, Mohamed Adel Serhani, Rachida Dssouli and Boualem Benatallahn (2017), “A Multi-Dimensional Trust Model for Processing Big Data over Competing Clouds", IEEE, pp.1-18.

[11] Sarbjeet Singh, Jagpreet Sidhu (2016), “Compliance-based Multidimensional Trust Evaluation System for determining trustworthiness of Cloud Service Providers”, Elsevier, pp. 109-132.

[12] Talal H. NoorQuan Z. ShengLina Yao. Schahram Dustdar, Anne H.H. Ngu (2016), "Cloud Armor: Supporting Reputation-Based Trust Management for Cloud Services”, IEEE, pp. 367-380.

[13] Z. Zheng, X. Wu, Y. Zhang, M. R. Lyu, and J. Wang (2013), “QoS ranking prediction for cloud services," IEEE Transactions on Parallel and Distributed Systems, vol. 24, no. 6, pp. 1213-1222.

[14] Rajendran, V.V.,Swamynathan,S., (2015), "Hybrid model for dynamic evaluation of trust in cloud services" Wireless Network, pp. $1-12$.

[15] Charband,Y.,Navimipour, N.J., (2016), “Online knowledge sharing mechanisms: a systematic review of the state of the art literature and recommendations for future research", Information of System.Front., pp. 1-21.

[16] Sidhu,J.,Singh,S., (2016), Improved TOPSIS method based trust evaluation framework for determining trustworthiness of cloud service providers, Journal of Grid Computing, pp. 1-25.

[17] Bahador Shojaiemehr, Amir Masoud Rahmani, Nooruldeen Nasih Qader (2018), "Cloud computing service negotiation: A systematic review”, Computer Standards \& Interfaces, Vol. 55, pp. 196-206,

\section{AUTHOR'S PROFILE}

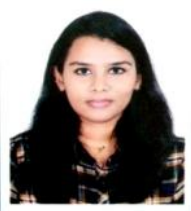

Venisha.A she has completed M.Tech Computer Science in SRM Institute of Science and Technology. And completed B.E Computer Science in TRP Engineering College, Trichy. She has completed herinternship in INTEL Banglore and worked in 5G Modem (Core Driver team) for one year.And done her UG and PG projects in Cloud Computing.

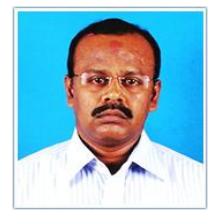

Dr.M.Murali he is currently working as Associate Professor in SRM Institute of Science and Technology.He did is Ph.D in SRMIST and did his M.Tech in Manonmaniam Sundaranar University.His research interest are Big Data,Data Mining,Machine Learning. He has 13 years of experience in Teaching.Member of ISTE,ISCA,IAENG,IET 